\title{
Integrin- $\beta 1$ regulates chondrocyte proliferation and apoptosis through the upregulation of GIT1 expression
}

\author{
LONG-QIANG ZHANG ${ }^{1,2^{*}}$, GUANG-ZONG ZHAO ${ }^{2 *}$, XIAO-YAN XU ${ }^{3}, \mathrm{JUN} \mathrm{FANG}^{2}$, \\ JING-MING CHEN ${ }^{2}$, JI-WEN LI ${ }^{2}$, XUE-JIAN GAO ${ }^{4}$, LI-JUAN HAO ${ }^{5}$ and YUN-ZHEN CHEN ${ }^{1}$
}

\author{
${ }^{1}$ Department of Orthopedics, Qilu Hospital of Shandong University, Jinan, Shandong 250012; ${ }^{2}$ Department of Orthopedics, \\ Yidu Central Hospital of Weifang, Weifang, Shandong 262500; ${ }^{3}$ Department of Oncology, Qingzhou Hospital of \\ Traditional Chinese Medicine, Qingzhou, Shandong 262500; ${ }^{4}$ Department of Orthopedics, \\ The 89th Hospital of PLA, Weifang, Shandong $261021 ;{ }^{5}$ Department of Urologic Surgery, \\ Yidu Central Hospital of Weifang, Weifang, Shandong 262500, P.R. China
}

Received November 7, 2014; Accepted February 5, 2015

DOI: $10.3892 /$ ijmm.2015.2114

\begin{abstract}
Chondrocytes play a critical role in the repair process of osteoarthritis, which is also known as degenerative arthritis. Integrins, as the key family of cell surface receptors, are responsible for the regulation of chondrocyte proliferation, differentiation, survival and apoptosis through the recruitment and activation of downstream adaptor proteins. Moreover, G-protein-coupled receptor kinase interacting protein-1 (GIT1) exerts its effects on cell proliferation and migration through interaction with various cytokines. It has been previously suggested that GIT1 acts as a vital protein downstream of the integrin-mediated pathway. In the present study, we investigated the effects of integrin- $\beta 1$ on cell proliferation and apoptosis, as well as the underlying mechanisms in chondrocytes in vitro. Following transfection with a vector expressing integrin- $\beta 1$, our results revealed that the overexpression of integrin- $\beta 1$ enhanced GIT1 expression, whereas the knockdown of integrin- $\beta 1$ by siRNA suppressed GIT1 expression. However, no significant effect was observed on integrin- $\beta 1$ expression following the enforced overexpression of GIT1, which suggests that GIT1 is localized downstream of integrin- $\beta 1$. In other words, integrin- $\beta 1$ regulates the expression of GIT1. Furthermore, this study demonstrated that integrin- $\beta 1$ and GIT1 increased the expression levels of aggrecan and type II collagen, thus promoting chondrocyte proliferation; however, they inhibited chondrocyte apoptosis. Taken together, our data demonstrate that integrin- $\beta 1$ plays a vital role in chondrocyte proliferation, differentiation
\end{abstract}

Correspondence to: Dr Yun-Zhen Chen, Department of Orthopedics, Qilu Hospital of Shandong University, 107 Wenhua West Road, Lixia, Jinan, Shandong 250012, P.R. China

E-mail: yzchen6501@hotmail.com

${ }^{*}$ Contributed equally

Key words: integrin- $\beta 1$, G-protein-coupled receptor kinase interacting protein-1, chondrocyte, proliferation and apoptosis. GIT1 exerts effects similar to those of integrin- $\beta 1$ and is a downstream target of integrin- $\beta 1$.

\section{Introduction}

Poorly treated or untreated articular cartilage defects easily give rise to osteoarthritis (OA), which is a progressive degradation of articular cartilage, resulting in the loss of joint function $(1,2)$. Cartilage cells play a crucial role in the repair process of OA. The investigation of the mechanisms responsible for the stimulation of chondrocyte proliferation and the suppression of chondrocyte apoptosis in vitro is of clinical significance. It has been previously demonstrated that a variety of cytokines, hormones, modifiers and drugs affect the biosynthesis of chondrocytes in in vitro experiments (3).

The interaction between chondrocytes and their surrounding extracellular matrix (ECM) has significant effects on the metabolic homeostasis of the articular cartilage (integrin- $\beta 1)(4,5)$. The ECM molecules, such as collagen, hyaluronan and aggrecan, are the most abundant macromolecules found in cartilage (3-5). Additionally, the overexpression of collagen and aggrecan has been found in the early stages of OA $(1,2)$. Integrins, cell surface adhesive proteins, are expressed in the majority of cells of multicellular animals, such as human chondrocytes $(4,5)$. Integrins are characterized by participating in cell-cell and cell-matrix communications. As transmembrane glycoprotein receptors, integrins also contribute to the cell attachment and cell signaling pathways. For instance, these adhesion proteins may affect bi-directional signaling in cells, affecting processes, such as cytoskeletal arrangement, growth factor signaling and gene transcription. Moreover, integrins mediate cell development, the immune response and diseases, such as cancer and autoimmune disorders. Integrins, as heterodimeric transmembrane glycoproteins, are made up of $\alpha$ and $\beta$ chains, being non-covalently bonded protein complexes $(4,5)$. The integrin- $\beta 1$ subfamily includes a variety of receptors, such as fibronectin, collagens and laminin for ECM proteins. The expression of target genes and the integrin expression and affinity may be altered by integrin binding due 
to cell signaling pathway stimulation $(5,6)$. Therefore, although the role of integrins in chondrocytes is not yet completely understood, it is believed that integrins exert significant effects on chondrocytes.

G-protein-coupled receptor kinase interacting protein-1 (GIT1) was originally identified by a yeast two-hybrid screen and is widely present in mammals and birds $(7,8)$. It is a scaffold protein, acting as a GTPase-activating protein for the ADP-ribosylation factor family of proteins (7-10). GIT1 is mainly located in cell adhesion and cytoplasmic complex structures and stimulates the activation of and the membrane recruitment of G-protein-coupled receptor kinase 2 (GRK2) (7). Initially, the identified function of GIT1 was to regulate cell surface G-protein-coupled receptors in cell transduction pathways (8). Later findings demonstrated that GIT1 plays a vital role in cell proliferation and migration through interaction with a variety of cytokines, including ARF, Rac1, Cdc42, GTPases, PAK, PIX, MEK1, PLC and paxillin $(9,10)$.

Thus far, it has been suggested that GIT1 exerts significant effects on chondrocytes. In the study by Menon et al (11), for example, GIT1 underwent Src-mediated tyrosine phosphorylation and was found to be a key component in phospholipase $C \gamma 2$ (PLC $\gamma 2$ ) phosphorylation, suggesting that it was a regulator of the receptor activator of NF- $\mathrm{KB}$ (RANK) signaling. Their results also demonstrated that GIT1 has great potential for use in the treatment of osteoporosis, due to its ability to regulate osteoclast function and bone mass (11). However, current studies on GIT1 in bone cells focus on the migration of osteoblasts. In the study by Ren et al (12), it was demonstrated that the phosphorylation of GIT1 tyrosine 321 is necessary for the association with focal adhesion kinase (FAK) and for the migration of osteoblasts induced by platelet-derived growth factor (PDGF). The results from the study by Liu et al (13) demonstrated that GIT1 and integrin $\alpha \mathrm{V} \beta 8$, in association with Pak-interacting exchange factor- $\beta$ $\beta$ PIX, had an effect on the regulation of vascular stability, cerebral angiogenesis and endothelial cell proliferation in the developing embryo. Furthermore, the data reported in the study by Sato et al (14) revealed that Src kinases transfer Rac-GEF $\beta P I X$ to the vicinity of the integrin through the tyrosine phosphorylation of GIT1, resulting in the regulation of the cytoskeletal reorganization downstream of $\alpha(\mathrm{IIb}) \beta 3$. As cell surface adhesion receptors, integrins are able to respond to a variety of stimuli from the extracellular environment in an attempt to regulate cell activity and function (6). Integrins, including $\alpha 1 \beta 1, \alpha 5 \beta 1$, $\alpha 10 \beta 1$ and $\alpha \mathrm{V} \beta 5$, are mainly expressed in the articular cartilage in healthy adults $(15,16)$. In addition, the study by Ren et al (17) provided evidence that periodic mechanical stress induces the activation of the integrin- $\beta 1-S r c-P L C \gamma 1 /$ Rac1-mediated extracellular signal-regulated kinase 1/2 (ERK1/2) mitogenic signaling pathway, enhancing chondrocyte proliferation and matrix synthesis. Taken together, these data suggest that there is a close association between GIT1 and integrins in bone cells. Therefore, the aim of this study was to examine the role of GIT1 and integrins (integrin- $\beta 1$ in particular) in chondrocytes.

\section{Materials and methods}

Cell culture. The dissection of 1-week-old Sprague-Dawley (SD) neonatal rats (Institute of Laboratory Animal Sciences, CAMS\&PUMC, Beijing, China) was performed in order to obtain limb joints under sterile conditions. Approximately $1 \mathrm{~mm}^{3}$ sections of cartilage were cut from the transparent central portion of the articular cartilage surface. The tissue sections were digested by $0.25 \%$ trypsin (Gibco, Beijing, China) at $37^{\circ} \mathrm{C}$ for $30 \mathrm{~min}$, and then centrifugation was performed at $1,400 \mathrm{rpm}$ for $5 \mathrm{~min}$ to collect the pellets. Following digestion of the pellets by $0.2 \%$ type II collagenase (Sigma, Beijing, China) at $37^{\circ} \mathrm{C}$ for $4 \mathrm{~h}$, the solution was filtered using a 200-mesh filter and centrifuged at 1,400 rpm for $5 \mathrm{~min}$. Furthermore, Dulbecco's modified Eagle's medium (DMEM)-F12 with $10 \%$ fetal bovine serum (FBS) (both from Gibco), penicillin (100 U/ml) and streptomycin $(50 \mathrm{U} / \mathrm{ml})$ were used to maintain the cells at $37^{\circ} \mathrm{C}$ in a humidified incubator with $5 \% \mathrm{CO}_{2}$ atmosphere. An inverted phase contrast microscope (CKX41-A32PH; Olympus, Shanghai, China) was used to observe the morphology of the purified cells after repeated isolation and culture. Subsequently, the cells were passed by $0.25 \%$ trypsin due to growing fully in the dish. The study protocol conformed with the Guide for the Care and Use of Laboratory Animals published by the US National Institutes of Health (NIH publication no. 85-23, revised 1985) and was approved by the Animal Care and Use Committee, Qilu Hospital of Shandong University, Jinan, China.

Vector construction and transfection with siRNA. RNA from chondrocytes was extracted using TRIzol reagent (Invitrogen, Shanghai, China). Following reverse transcription, polymerase chain reaction (PCR) was used to amplify the integrin- $\beta 1$ and GIT1 coding regions. They were then cloned into the pcDNA3.1 vector (Promega, Beijing, China) through the KpnI and EcoRI (Takara, Dalian, China) restriction enzymes. The gene amplification primers are listed in Table I. Subsequently, $1 \times 10^{5}$ cells $/ \mathrm{ml}$ cells were seeded in 6 -well plates and incubated for $24 \mathrm{~h}$. Plasmid and siRNA transfection was carried out using Lipofectamine 2000 (Invitrogen) following the manufacturer's instructions, when the cell confluence reached approximately $70 \%$. Integrin- $\beta 1$ siRNA (target sequence 5'-AAA AGT CTT GGA ACA GAT CTG-3') and negative control (NC) siRNA (HP GenomeWide siRNA; Qiagen, Hilden, Germany) were prepared and transfected at $100 \mathrm{nM}$ for $48 \mathrm{~h}$ as previously described (18). Subsequently, the transfected cells were analyzed by quantitative PCR and western blot analysis.

Quantitative fluorescence PCR. The cells were collected, and total RNA was extracted using TRIzol reagent. Reverse transcription was performed with $1 \mu \mathrm{g}$ of RNA following quantification. Quantitative PCR was conducted using SYBR-Green (Toyobo, Osaka, Japan) with $100 \mathrm{ng}$ of cDNA in a $20 \mu \mathrm{l}$ system. The primer sequences are listed in Table I. The reaction conditions were as follows: $95^{\circ} \mathrm{C}$ for $5 \mathrm{~min}$, followed by 40 cycles of $95^{\circ} \mathrm{C}$ for $30 \mathrm{sec}, 55^{\circ} \mathrm{C}$ for $30 \mathrm{sec}$, and $72^{\circ} \mathrm{C}$ for $30 \mathrm{sec}$. All reactions were run in triplicate, and quantitative analysis was performed by comparing the $2^{-\Delta \Delta \mathrm{Ct}}$ values.

Western blot analysis. Total cellular protein was extracted from the cells using lysis buffer. The supernatant containing proteins was obtained after shaking at $4^{\circ} \mathrm{C}$ for $20 \mathrm{~min}$ and centrifugation at $14,000 \mathrm{rpm}$ at $4^{\circ} \mathrm{C}$ for $10 \mathrm{~min}$. The Bradford protein assay (Pierce, Rockford, IL, USA) was used to determine the protein concentration. An equal amount of proteins 
Table I. Sequences of primers used for gene amplification and PCR.

Name

Sense $\left(5^{\prime} \rightarrow 3^{\prime}\right)$

Antisense $\left(5^{\prime} \rightarrow 3^{\prime}\right)$

Primer sets for CdS

amplification of integrin- $\beta 1$

GIT1

Primer sets for $\mathrm{qPCR}$

$\beta$-actin

Integrin- $\beta 1$

GIT1

Aggrecan

Type II collagen

\section{GGGGTACCGCCACCATGAATTTGCA ACTGGTTTTC \\ GGGGTACCGCCACCATGTCCCG GAAGGGGCCG \\ CGGAATTCTCATTTTCCCT CATACTTCGG \\ CGGAATTCTCACTGTTTCTTC TCTCGGGTGG}

GTCCACCTTCCAGCAGATG
TTACAAGAGTGCCGTGACAACTG
ATGGATGTGTATGACGAAGTG
AACAGCATCTACCAAGACAAG
CTCAAGTCGCTGAACAAC

$\begin{array}{lr}\text { CTCAGTAACAGTCCGCCTAG } & 98 \\ \text { GACTAAGATGCTGCTGCTGTGAG } & 106 \\ \text { GGTTGACTGGCAGGAAGG } & 115 \\ \text { GCATCACTTCACACCGATAG } & 121 \\ \text { CAGTAGTCTCCGCTCTTC } & 122\end{array}$

CdS, coding sequence; GIT1, G-protein-coupled receptor kinase interacting protein-1. Bold and italics letters represent the restriction enzyme cut site and its protective base; underlined letters represent the Kozak sequence.

was loaded on $8 \%$ glycine gels [sodium dodecyl sulfatepolyacrylamide electrophoresis (SDS-PAGE)]. Following electrophoresis, the separate proteins were transferred onto nitrocellulose membranes (Pierce). Subsequently, 5\% non-fat milk (Pierce) in TBST buffer was used to block the membranes for $1 \mathrm{~h}$. Subsequently, the membranes were incubated with GIT1 (\#2919; Cell Signaling Technology, Beverly, MA, USA), integrin- $\beta 1$ (\#9699; Cell Signaling Technology), aggrecan (sc-25674; Santa Cruz Biotechnology, Santa Cruz, CA, USA), type II collagen (sc-28887; Santa Cruz Biotechnology) and $\beta$-actin (\#8457; Cell Signaling Technology) primary antibodies at 1:1,000 dilutions overnight at $4^{\circ} \mathrm{C}$, and anti-rabbit IgG monoclonal antibody conjugated with horseradish peroxidase ( $\# 5127$; Cell Signaling Technology) was then added at a 1:7,000-8,000 dilution followed by incubation for $1 \mathrm{~h}$ at $37^{\circ} \mathrm{C}$. TBST was used for washing the membranes every $10 \mathrm{~min}$, for a total of $30 \mathrm{~min}$. Protein bands were detected by ECL (Pierce). The light-emitting film was scanned using a gel imaging system (UVP Co., Upland, CA, USA) of western blot analysis and gray values of the band were measured using Gel-Pro Analyzer software (Media Cybernetics, Inc., Silver Spring, MD, USA).

BrdU cell proliferation assay. BrdU assay was used to investigate the role of integrin- $\beta 1$ and GIT1 in cell proliferation. Briefly, the cultured cells were seeded in 6-well plates and incubated for $24 \mathrm{~h}$, and then plasmid and siRNA transfection was carried out using Lipofectamine 2000. A BrdU colorimetric immunoassay kit (Cell Proliferation ELISA; Roche Diagnostics GmbH, Mannheim, Germany) was used for the quantification of cell proliferation according to the protocol provided by the manufacturer. Cell proliferation was expressed as the mean percentage of the control values (set at 100\%).

TUNEL-DAPI co-staining assay. TUNEL-DAPI co-staining assay with the In Situ Cell Death Detection kit (Roche Diagnostics $\mathrm{GmbH}$ ) was used for measuring cell apoptosis. Briefly, after being washed with phosphate-buffered saline (PBS), the cultured cells were fixed with $4 \%$ formaldehyde at $4^{\circ} \mathrm{C}$ for $25 \mathrm{~min}$. The cells were incubated with $0.2 \%$ Triton $\mathrm{X}-100$ for $5 \mathrm{~min}$. Following incubation with $100 \mu \mathrm{l}$ equilibration buffer at room temperature for $10 \mathrm{~min}$, the cells were mixed with $50 \mu \mathrm{l}$ TUNEL reaction mixture containing nucleotide mixture and terminal deoxynucleotidyl transferase (TdT) for $60 \mathrm{~min}$ at $37^{\circ} \mathrm{C}$. Subsequently, $2 \mathrm{X}$ saline sodium citrate (SSC) was used to wash the cells for $15 \mathrm{~min}$. The cells were then incubated with $0.3 \% \mathrm{H}_{2} \mathrm{O}_{2}$ for $10 \mathrm{~min}$ and streptavidin working solution for $30 \mathrm{~min}$ at room temperature and $0.5 \mu \mathrm{g} / \mathrm{ml}$ of DAPI were then added and the cells were incubated a humidified chamber in the dark for $5 \mathrm{~min}$ at room temperature. Finally, the cells were examined and photographed under a fluorescence microscope (Nikon Eclipse 80i; Nikon, Tokyo, Japan).

Statistical analysis. The experiments were carried out at least in triplicate, and the results are expressed as the means \pm SD. The SPSS statistical package (SPSS 13.0 for Windows; SPSS, Inc., Chicago, IL, USA) was used for statistical analysis. Differences between 2 groups were analyzed using a two-tailed Student's t-test and those between 3 or more groups by one-way ANOVA multiple comparisons. Values of $\mathrm{P}<0.05$ or $\mathrm{P}<0.01$ were considered to indicate statistically significant differences.

\section{Results}

Integrin- $\beta 1$ increases the expression of GIT1. In the present study, siRNA silencing techniques, quantitative PCR and western blot analysis were used to analyze the role of integrin- $\beta 1$, GIT1 and their crosstalk in cartilage cells. The cells were seeded in 6-well plates and incubated for $24 \mathrm{~h}$, and then siRNA transfection was carried out using Lipofectamine 2000. Subsequently, quantitative PCR and western blot analysis were carried out to detect the mRNA and protein expression levels. Following transfection, the overexpression of integrin- $\beta 1$ was found to promote GIT1 expression, whereas the silencing of integrin- $\beta 1$ by siRNA inhibited the expression of GIT1 (Fig. 1). However, GIT1 overexpression 

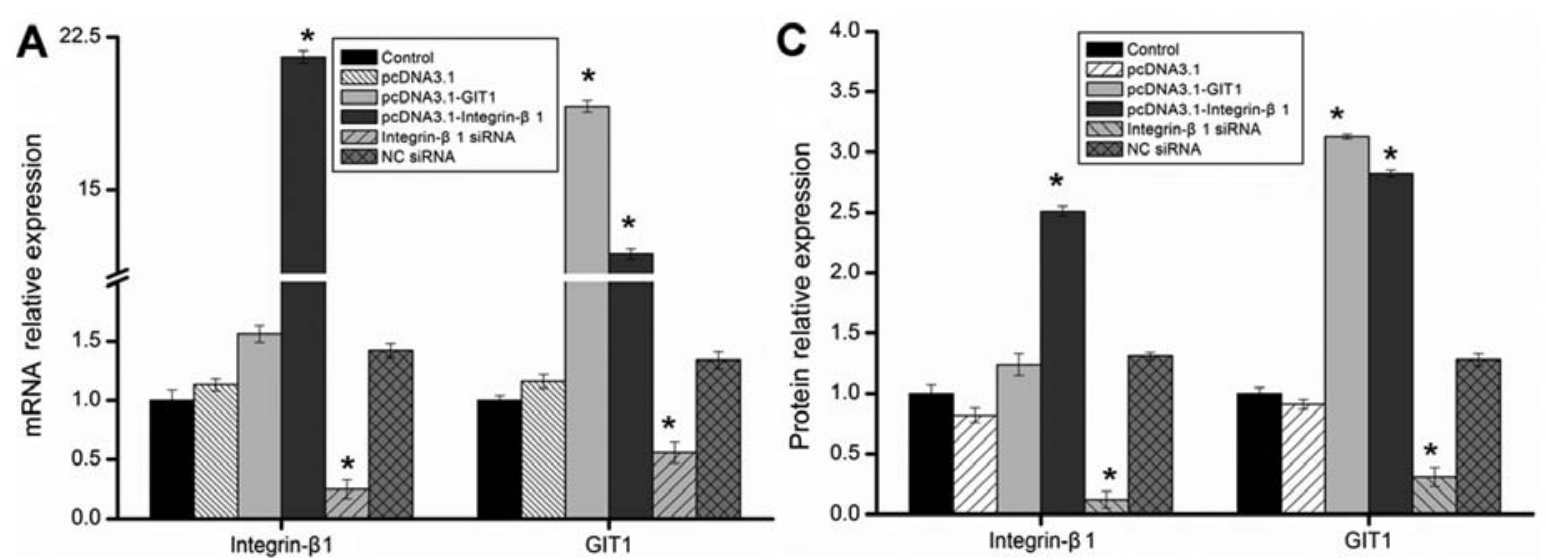

B Control pcDNA3.1 pcDNA3.1 pcDNA3.1 Integrin- $\beta 1$ NC siRNA

GIT1 Integrin- $\beta 1 \quad$ siRNA

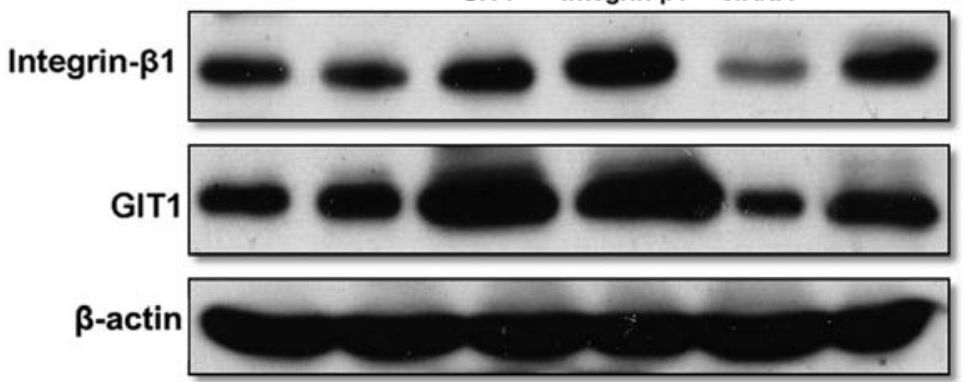

Figure 1. The expression of G-protein-coupled receptor kinase interacting protein-1 (GIT1) is promoted by integrin- $\beta 1$. The cells were seeded in 6-well plates and incubated for $24 \mathrm{~h}$, and then integrin- $\beta 1$ siRNA transfection was carried out using Lipofectamine 2000. Following siRNA transfection, the expression of integrin- $\beta 1$ and GIT1 was measured by (A) quantitative fluorescence PCR and (B and C) western blot analysis. The experiments were carried out at least in triplicate, and the results are expressed as the means $\pm \mathrm{SD},{ }^{*} \mathrm{P}<0.05$ vs. the control.

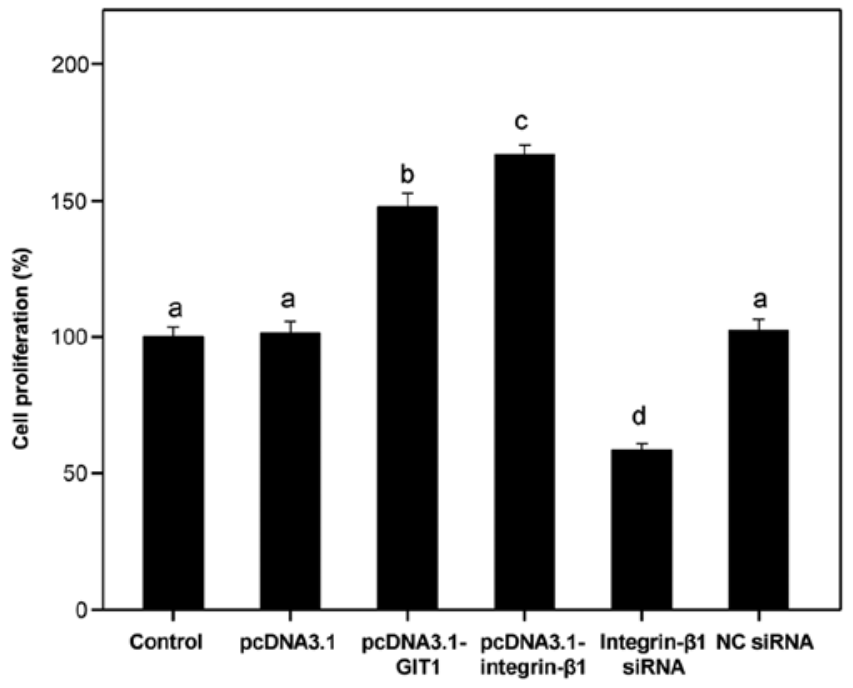

Figure 2. Chondrocyte proliferation is affected by integrin- $\beta 1$ and G-proteincoupled receptor kinase interacting protein-1 (GIT1). PcDNA3.1 was the vector used for integrin- $\beta 1$ and GIT1 transfection. The cells were seeded in 6-well plates and incubated for $24 \mathrm{~h}$, and then integrin- $\beta 1$ and GIT1 siRNA transfection was carried out using Lipofectamine 2000. BrdU assay was used for the examination of cell proliferation; bars labeled with diffent letters (a-d) indicate a statistically significant difference between groups $(\mathrm{P}<0.01)$.

had no significant effect on the expression of integrin- $\beta 1$ (Fig. 1). These results suggest that integrin- $\beta 1$ regulates the expression of GIT1 and is located upstream of GIT1.
Integrin- $\beta 1$ and GIT1 promote chondrocyte proliferation. The effects of integrin- $\beta 1$ and GIT1 on chondrocyte proliferation were also investigated. Briefly, the cells were seeded into 6-well plates and incubated for $24 \mathrm{~h}$, and then integrin- $\beta 1$-pcDNA3.1, GIT1-pcDNA3.1 and integrin- $\beta 1$ siRNA transfection was carried out using Lipofectamine 2000. BrdU assay was used to assess the proliferation of cartilage cells. The results revealed that the overexpression of integrin- $\beta 1$ and GIT1 enhanced chondrocyte proliferation, although the effects of integrin- $\beta 1$ overexpression on chondrocyte proliferation were more prominent (Fig. 2). In other words, integrin- $\beta 1$ plays an essential role in the regulation of chondrocyte proliferation. When integrin- $\beta 1$ expression was depleted using siRNA, chondrocyte proliferation was significantly inhibited. These results suggest that integrin- $\beta 1$ plays a role in chondrocyte proliferation.

Integrin- $\beta 1$ and GIT1 affect the synthesis of ECM in chondrocytes. It is well known that aggrecan and type II collagen are abundant in cartilage ECM (3-5); thus, the synthesis of the ECM can be determined using them as references. As a result, the activity and function of tissue engineering are reflected by chondrocyte proliferation and the gene expression levels of type II collagen and aggrecan. Western blot analysis was used to investigate the protein expression levels. The expression levels of aggrecan and type II collagen were significantly enhanced by the overexpression of integrin- $\beta 1$ and GIT1 (Fig. 3). However, the effects of integrin- $\beta 1$ on the stimulation of the expression of aggrecan and type II collagen were much more prominent than 

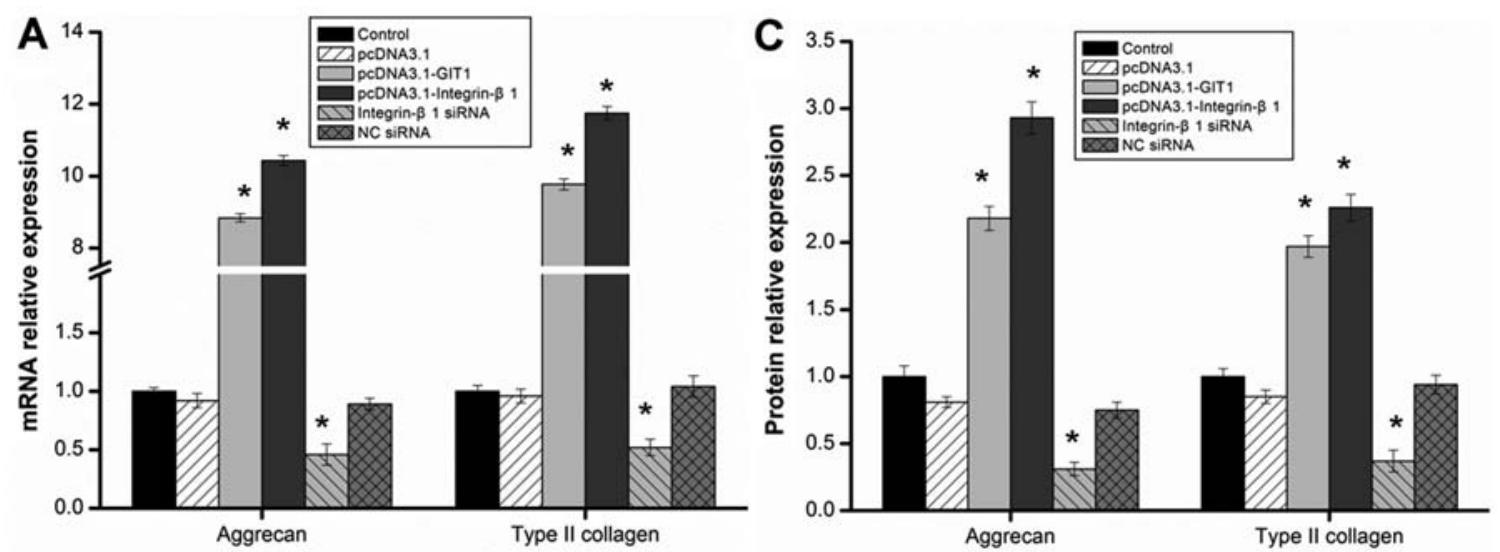

B

Control pcDNA3.1 pcDNA3.1 pcDNA3.1 Integrin- $\beta 1$ NC siRNA GIT1 Integrin- $\beta 1$ siRNA

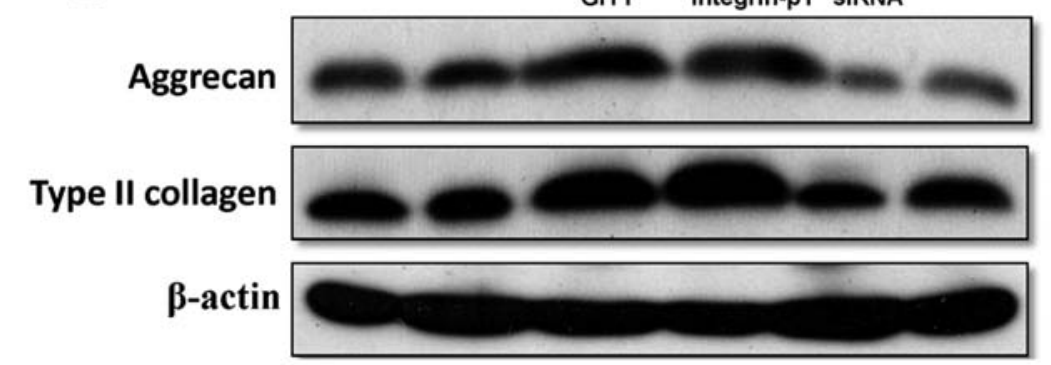

Figure 3. Synthesis of extracellular matrix in chondrocytes is regulated by integrin- $\beta 1$ and G-protein-coupled receptor kinase interacting protein-1 (GIT1). The cells were seeded in 6-well plates and incubated for $24 \mathrm{~h}$, and then siRNA transfection was carried out using Lipofectamine 2000. The expression levels of aggrecan and type II collagen were examined by (A) quantitative fluorescence PCR and (B and C) western blot analysis. Experiments were carried out at least in triplicate, and the results are expressed as the means $\pm \mathrm{SD},{ }^{*} \mathrm{P}<0.05$ vs. the control.

those of GIT1 (Fig. 3). On the other hand, the expression levels of aggrecan and type II collagen were significantly reduced when integrin- $\beta 1$ expression was depleted using specific siRNA (Fig. 3). Therefore, these results demonstrate that integrin- $\beta 1$ regulates aggrecan and type II collagen expression.

Integrin- $\beta 1$ inhibits chondrocyte apoptosis. Programmed cell death (PCD), known as apoptosis, is a crucial process in multicellular organisms. Chondrocyte apoptosis plays a vital role in bone remodeling. In the present study, in order to further examine the role of integrin- $\beta 1$ in cartilage cells, TUNEL-DAPI assay was used to measure DNA fragmentation resulting from apoptotic signaling cascades. For the purpose of understanding the role of integrin- $\beta 1$ in chondrocyte apoptosis, TUNEL assay was used to identify the presence of nicks in the DNA. DNA fragmentation, which is associated with apoptotic events, was observed in the chondrocytes (Fig. 4). The results revealed that the occurrence of apoptotic cell death was significantly suppressed by the overexpression of integrin- $\beta 1$ (Fig. 4). Moreover, following the silencing of integrin- $\beta 1$ using specific siRNA, there were no significant effects observed on cell apoptosis (Fig. 4). These results suggest that integrin- $\beta 1$ is required for the survival of chondrocytes.

\section{Discussion}

Integrins are a key family of cell surface adhesion receptors regarded as heterodimeric transmembrane glycoproteins (19). It has been demonstrated that integrins are widely expressed in cells. There are several integrins located on the cell surface of the majority the cells, playing a critical role in a variety of metabolic activities $(19,20)$. In fact, integrins without any kinase activity regulate the recruitment and activation of the downstream adapter protein of integrin signaling, resulting in intracellular signal transduction $(18,19)$.

Integrins function as a bridge connecting chondrocytes and ECM, regulating the interaction between the two, thus affecting the differentiation, proliferation, growth and apoptosis of cartilage cells and ECM structure (3-5). It is known that integrins, including integrins $\alpha 1 \beta 1, \alpha 2 \beta 1, \alpha 3 \beta 1, \alpha 5 \beta 1$, $\alpha 6 \beta 1, \alpha 10 \beta 1, \alpha \mathrm{V} \beta 3$ and $\alpha \mathrm{V} \beta 5$ in chondrocytes play a role in mechanical signal transduction (of which integrins $\alpha 1 \beta 1, \alpha 3 \beta 1$, $\alpha 5 \beta 1$ play the most prominent role) and that integrin $\alpha 10 \beta 1$ is a main molecule for stabilizing the chondrocyte phenotype (18). Integrin- $\beta 1$ is responsible for cartilage tissue repair and remodeling by binding and assembling important structural ECM proteins, such as collagen. For instance, integrins $1 \beta 1$ and $\alpha 2 \beta 1$ have been found to mediate chondrocyte adhesion to type II collagen and laminin $(3-5,18)$. However, integrins $\alpha 3 \beta 1$ and $\alpha 5 \beta 1$ mediate the adhesion of cartilage cells to type II collagen and fibronectin (19). In addition, Schagemann et al (20) found that biological materials can promote chondrocyte CD44 and integrin- $\beta 1$ expression, being conducive to cartilage repair and reconstruction in the investigation of tissue engineered cartilage.

In the present study, we demonstrated that the upregulation of integrin- $\beta 1$ promoted chondrocyte proliferation and reduced apoptosis. Moreover, in the study by Wang et al (21), it was suggested that integrins play an essential role in the regulation of chondrocyte proliferation and differentiation, and that 

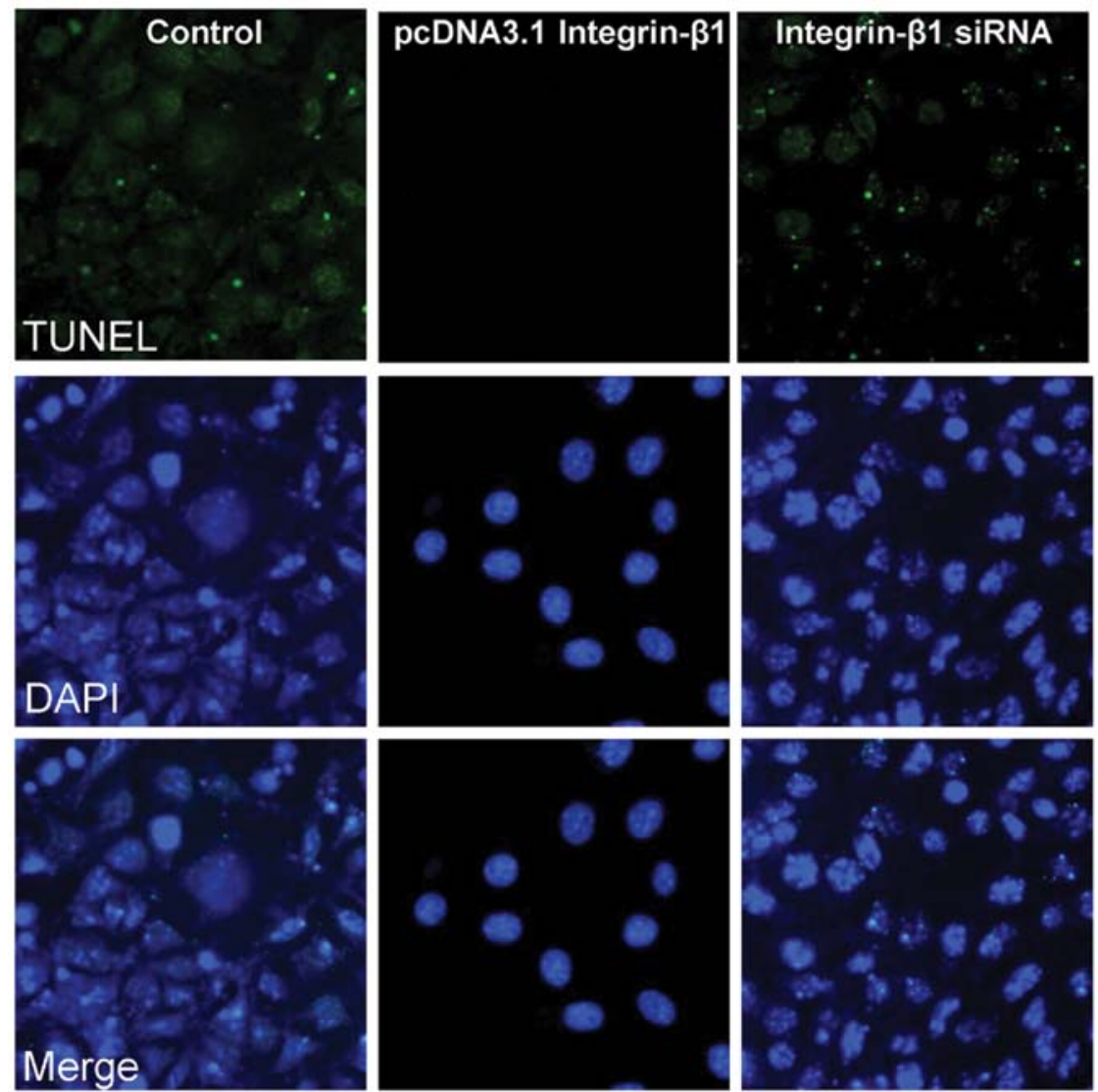

Figure 4. Chondrocyte apoptosis is affected by integrin- $\beta 1$. The cells were seeded in 6 -well plates and incubated for $24 \mathrm{~h}$, and then integrin- $\beta 1$ siRNA transfection was carried out using Lipofectamine 2000. TUNEL-DAPI co-staining assay was used to examine cell apoptosis.

RhoA is characterized as the downstream target of integrin molecules. Fibroblast growth factor (FGF) regulates the proliferation of chondrocytes depending on cell adhesion between fibronectin and integrin- $\alpha 5 \beta 1$ (22). Our data also showed that integrin- $\beta 1$ inhibited chondrocyte apoptosis. In the study by Cao et al (23), it was demonstrated that integrin- $\beta 1$-mediated collagen-chondrocyte interactions protect chondrocytes from apoptosis. The results of the study by Lee et al (24) indicated that mechanical stimulation increased osteoblast proliferation, mediating the regulation by integrin- $\beta 1$ and integrin- $\beta 3$ signaling. Furthermore, the overexpression of integrin- $\beta 1$ enhanced the expression levels of aggrecan and type II collagen for the purpose of maintaining the differentiated phenotype of chondrocytes. Most importantly, type II collagen and aggrecan are the major ECM components responsible for maintaining the phenotypic stability of chondrocytes (3-5). Chondrocyte metabolism is closely associated with collagen and proteoglycan synthesis and decomposition. For instance, type II collagen is the major collagen found in cartilage, accounting for $90-95 \%$ of total collagen (25). The qualitative and quantitative changes in type II collagen and proteoglycan synthesis are the direct cause of articular cartilage losing its normal biomechanical properties, having a close association with OA $(25,26)$. The results of the study by Bouchet et al (27) demonstrated that the overexpression of integrin- $\beta 1$ in cartilage cells had an effect on the maintenance of the chondrocytic phenotype.

GIT1, a scaffold protein, is responsible for PLC $\gamma$ and ERK1/2 signaling induced by angiotensin II and epidermal growth factor (7-10). According to our results from western blot analysis and siRNA depletion, GIT1 was shown to be a downstream molecule of integrin- $\beta 1$. In other words, it was shown that integrin- $\beta 1$ regulated the expression of GIT1, but the upregulation of GIT1 had little effect on the expression of integrin- $\beta 1$. Moreover, our results demonstrated that GIT1 overexpression had an effect similar to that of integrin- $\beta 1$ overexpression on the promotion of chondrocyte proliferation and differentiation, as well as the suppression of chondrocyte apoptosis. However, to date, there has been little research focusing on GIT1 expression in cartilage cells, although studies have focused on GIT1 expression in other types of cells. For example, in the study by Rui et al (28), it was demonstrated that GIT1Y321 phosphorylation is required for ERK1/2- and PDGF-dependent VEGF secretion from osteoblasts to promote angiogenesis and bone healing. In their study, Pang et al (29) demonstrated that the depletion of GIT1 markedly inhibited intima formation following carotid ligation, leading to the reduction of vascular smooth muscle cell (VSMC) proliferation and the stimulation of apoptosis. Additionally, GIT1 exerts effects on vascular remodeling through the PLC $\gamma$ - and ERK1/2-mediated VSMC proliferation, migration and regulation of apoptosis (29). In another study by Pang et al (30), it was revealed that in global GIT1 knockout mice, cardiomyocyte apoptosis and cardiac dysfunction were enhanced, suggesting the role of GIT1 in post-natal cardiac maturation by mitochondrial biogenesis and function regulation. The results of the study by Zhang et al (31) indicated that cell apoptosis was reduced by GIT through the 
inositol 1,4,5-trisphosphate receptor [IP(3)R]-mediated $\mathrm{Ca}(2+)$ signaling pathway. Therefore, GIT1 plays a role in the regulation of cell proliferation and apoptosis.

In conclusion, as shown by our results, integrin- $\beta 1$ exerts a significant effect on the proliferation, apoptosis and differentiation of chondrocytes. More specifically, integrin- $\beta 1$ stimulates chondrocyte proliferation and differentiation, and suppresses chondrocyte apoptosis by increasing the expression of GIT1. GIT1, as a downstream effector of integrin- $\beta 1$, exerts similar effects on the proliferation, apoptosis and differentiation of chondrocytes. However, the role of integrin- $\beta 1$ and its underlying mechanisms of action in regulating the proliferation, differentiation and apoptosis of cartilage cells remain elusive. Therefore, further research is required to provide more compelling evidence of the effects of integrin- $\beta 1$ on chondrocytes.

\section{References}

1. Fernandes AM, Herlofsen SR, Karlsen TA, Küchler AM, Fløisand Y and Brinchmann JE: Similar properties of chondrocytes from osteoarthritis joints and mesenchymal stem cells from healthy donors for tissue engineering of articular cartilage. PLoS One 8: e62994, 2013.

2. Dreier R: Hypertrophic differentiation of chondrocytes in osteoarthritis: the developmental aspect of degenerative joint disorders. Arthritis Res Ther 12: 216, 2010.

3. Tanaka Y, Ogasawara T, Asawa Y, Yamaoka H, Nishizawa S, Mori Y, Takato T and Hoshi K: Growth factor contents of autologous human sera prepared by different production methods and their biological effects on chondrocytes. Cell Biol Int 32: 505-514, 2008

4. Lowell CA and Mayadas TN: Overview: studying integrins in vivo. Methods Mol Biol 757: 369-397, 2012.

5. Brakebusch C, Bouvard D, Stanchi F, Sakai T and Fassler R: Integrins in invasive growth. J Clin Invest 109: 999-1006, 2002.

6. Spiteri C, Raizman I, Pilliar RM and Kandel RA: Matrix accumulation by articular chondrocytes during mechanical stimulation is influenced by integrin-mediated cell spreading. J Biomed Mater Res A 94: 122-129, 2010.

7. Premont RT, Claing A, Vitale N,Freeman JL, Pitcher JA, Patton WA, Moss J, Vaughan M and Lefkowitz RJ: beta2-Adrenergic receptor regulation by GIT1, a G protein-coupled receptor kinase-associated ADP ribosylation factor GTPase-activating protein. Proc Natl Acad Sci USA 95: 14082-14087, 1998.

8. Claing A, Chen W, Miller WE, Vitale N, Moss J, Premont RT, Lefkowitz RJ: beta-Arrestin-mediated ADP-ribosylation factor 6 activation and beta 2-adrenergic receptor endocytosis. J Biol Chem 276: 42509-42513, 2001.

9. Hsu RM, Tsai MH, Hsieh YJ, Lyu PC and Yu JS: Identification of MYO18A as a novel interacting partner of the PAK2/betaPIX/GIT1 complex and its potential function in modulating epithelial cell migration. Mol Biol Cell 21: 287-301, 2010.

10. Gavina M, Za L, Molteni R, Pardi R and de Curtis I: The GIT-PIX complexes regulate the chemotactic response of rat basophilic leukaemia cells. Biol Cell 102: 231-244, 2010.

11. Menon P, Yin G, Smolock EM, Zuscik MJ, Yan C and Berk BC: GPCR kinase 2 interacting protein 1 (GIT1) regulates osteoclast function and bone mass. J Cell Physiol 225: 777-785, 2010.

12. Ren Y, Yu L, Fan J, Rui Z, Hua Z, Zhang Z, Zhang N and Yin G: Phosphorylation of GIT1 tyrosine 321 is required for association with FAK at focal adhesions and for PDGF-activated migration of osteoblasts. Mol Cell Biochem 365: 109-118, 2012.

13. Liu J, Zeng L, Kennedy RM, Gruenig NM and Childs SJ: $\beta$ Pix plays a dual role in cerebral vascular stability and angiogenesis, and interacts with integrin $\alpha v \beta 8$. Dev Biol 363: 95-105, 2012.
14. Sato H, Suzuki-Inoue K, Inoue O and Ozaki Y: Regulation of adaptor protein GIT1 in platelets, leading to the interaction between GIT1 and integrin alpha(IIb)beta3. Biochem Biophys Res Commun 368: 157-161, 2008.

15. Loeser RF, Sadiev S, Tan L and Goldring MB: Integrin expression by primary and immortalized human chondrocytes: evidence of a differential role for alphalbeta1 and alpha2beta1 integrins in mediating chondrocyte adhesion to types II and VI collagen. Osteoarthritis Cartilage 8: 96-105, 2000.

16. Gigout A, Jolicoeur M, Nelea M, Raynal N, Farndale R and Buschmann MD: Chondrocyte aggregation in suspension culture is GFOGER-GPP- and betal integrin-dependent. J Biol Chem 283: 31522-31530, 2008.

17. Ren K, Ma Y, Huang Y, Liang W, Liu F, Wang Q, Cui W, Liu Z, Yin G and Fan W: Periodic mechanical stress activates MEK1/2-ERK1/2 mitogenic signals in rat chondrocytes through Src and PLC $\gamma 1$. Braz J Med Biol Res 44: 1231-1242, 2011.

18. Gohlke H, Schmitz B, Sommerfeld A, Reinehr R and Haussinger D: $\alpha 5 \beta 1$-integrins are sensors for tauroursodeoxycholic acid in hepatocytes. Hepatology 57: 1117-1129, 2013.

19. Kim SH, Turnbull J and Guimond S: Extracellular matrix and cell signalling: the dynamic cooperation of integrin, proteoglycan and growth factor receptor. J Endocrinol 209: 139-151, 2011.

20. Schagemann JC1, Kurz H, Casper ME, Stone JS, Dadsetan M, Yu-Long S, Mrosek EH, Fitzsimmons JS, O'Driscoll SW and Reinholz GG: The effect of scaffold composition on the early structural characteristics of chondrocytes and expression of adhesion molecules. Biomaterials 31: 2798-2805, 2010.

21. Wang G, Woods A, Sabari S, Pagnotta L, Stanton LA and Beier F: RhoA/ROCK signaling suppresses hypertrophic chondrocyte differentiation. J Biol Chem 279: 13205-13214, 2004.

22. Enomoto-Iwamoto M, Iwamoto M, Nakashima K, Mukudai Y, Boettiger D, Pacifici M, Kurisu K and Suzuki F: Involvement of alpha5betal integrin in matrix interactions and proliferation of chondrocytes. J Bone Miner Res 12: 1124-1132, 1997.

23. Cao L, Lee V, Adams ME, Kiani C, Zhang Y, Hu W and Yang BB: beta-Integrin-collagen interaction reduces chondrocyte apoptosis. Matrix Biol 18: 343-355, 1999.

24. Lee DY, Li YS, Chang SF, Zhou J, Ho HM, Chiu JJ and Chien S: Oscillatory flow-induced proliferation of osteoblast-like cells is mediated by alphavbeta 3 and beta1 integrins through synergistic interactions of focal adhesion kinase and She with phosphatidylinositol 3-kinase and the Akt/mTOR/p70S6K pathway. J Biol Chem 285: 30-42, 2010.

25. Poole AR, Nelson F, Dahlberg L, et al: Proteolysis of the collagen fibril in osteoarthritis. Biochem Soc Symp 70: 115-123, 2003.

26. Henrotin Y, Addison S, Kraus V and Deberg M: Type II collagen markers in osteoarthritis: what do they indicate? Curr Opin Rheumatol 19: 444-450, 2007.

27. Bouchet BY, Colon M, Polotsky A, Shikani AH, Hungerford DS and Frondoza CG: Beta-1 integrin expression by human nasal chondrocytes in microcarrier spinner culture. J Biomed Mater Res 52: 716-724, 2000.

28. Rui Z, Li X, Fan J, Ren Y, Yuan Y, Hua Z, Zhang N and Yin G: GIT1Y321 phosphorylation is required for ERK1/2- and PDGF-dependent VEGF secretion from osteoblasts to promote angiogenesis and bone healing. Int J Mol Med 30: 819-825, 2012.

29. Pang J, Xu X, Wang X, Majumder S, Wang J, Korshunov VA and Berk BC: G-protein-coupled receptor kinase interacting protein-1 mediates intima formation by regulating vascular smooth muscle proliferation, apoptosis, and migration. Arterioscler Thromb Vasc Biol 33: 999-1005, 2013.

30. Pang J, Xu X, Getman MR, Shi X, Belmonte SL, Michaloski H, Mohan A, Blaxall BC and Berk BC: G protein coupled receptor kinase 2 interacting protein 1 (GIT1) is a novel regulator of mitochondrial biogenesis in heart. J Mol Cell Cardiol 51: 769-776, 2011.

31. Zhang S, Hisatsune C, Matsu-Ura T and Mikoshiba K: G-proteincoupled receptor kinase-interacting proteins inhibit apoptosis by inositol 1,4,5-triphosphate receptor-mediated $\mathrm{Ca}^{2+}$ signal regulation. J Biol Chem 284: 29158-29169, 2009. 